\title{
Prediction of short term developmental outcomes in preterm infants utilizing cranial ultrasound examination.
}

\author{
Huynh Quang Huy ${ }^{*}$, Ton Nu Van Anh², Nguyen Huu Son ${ }^{3}$, Tran Thi Thuy Quynh ${ }^{4}$ \\ ${ }^{1}$ Department of Radiology, Pham Ngoc Thach University of Medicine and HCMC Oncology Hospital, Vietnam \\ ${ }^{2}$ Department of Pediatrics, Hue University of Medicine and Pharmacy, Hue University, Vietnam \\ ${ }^{3}$ Department of Pediatrics, Pediatric Center-Hue Central Hospital, Vietnam \\ ${ }^{4}$ Department of Pediatrics, Quang Ngai Hospital for Children and Women, Vietnam
}

\begin{abstract}
Background: Premature birth is associated with brain lesions mostly resulting from hypoxia-ischaemia and haemorrhage. Clinicians routinely need to provide parents and carers with prognostic information for their vulnerable infants, and most do this with the aid of some forms of neuroimaging. Studies of the relationship between ultrasound images from preterm newborns and developmental delay at 6 months' corrected age.

Methods: We evaluated associations between ultrasound-defined lesions of the brain and developmental delays at 6 months' corrected age in 79 children born before the 37th postmenstrual week. Brain ultrasound scans were read for concordance on 4 lesions: Intraventricular haemorrhage; Periventricular leukomalacia; Ventricular dilatation; Other lesion (congenital anomaly, cystic lesion). Developmental assessment at 6 months by using Denver II developmental screening test and a neurologic examination.

Results: Fully $24.1 \%$ of all of the children had delayed mental and/or psychomotor development. Ultrasound abnormalities were more strongly associated with developmental delays. They were intraventricular haemorrhage (RR 9.6) had periventricular leukomalacia (RR 10.3), ventricular dilatation (RR 18), congenital anomaly (RR 18) and cystic lesion (RR 18). Several peninatal variables including afgar score at 5 mins $<7$, hyaline membrane disease and mechanical ventilation are each associated with an elevated risk of developmental delays.

Conclusion: Cranial ultrasound provides a good screening tool for detection of brain injury in preterm infants during hospitalisation. Future studies are necessary to evaluate whether MRI of preterm infants can lead to a more accurate prediction of the neurodevelopmental impairment.
\end{abstract}

Keywords: Cranial ultrasound, Preterm newborn, Developmental delay.

Abbreviations: cUS: Cranial Ultrasound; RR: Relative Risk; MRI: Resonance Imaging; CT: Computerized Tomography; GA: Gestational Age; IVH: Intraventricular Haemorrhage; PVL: Periventricular Leukomalacia; GMH: Germinal Matrix Hemorrhage.

Accepted on May 25, 2019

\section{Introduction}

Premature birth is associated with brain lesions mostly resulting from hypoxia-ischaemia and haemorrhage $[1,2]$. Compared with full-term infants, preterm infants are in a state of continuous and immature brain development, are more vulnerable to injury and long-term disability, and are at high risk of death. Preterm birth is increasing and the rate of developmental impairment in survivors remains high [3-5]. Clinicians routinely need to provide parents and carers with prognostic information for their vulnerable infants, and most do this with the aid of some forms of neuroimaging. Various imaging techniques, mainly ultrasound (US) and magnetic resonance imaging (MRI), have been used for this purpose $[1,2]$.

US is the most widely used cranial imaging modality in the neonatal intensive care unit. US machines are portable, the images can be acquired at bedside, and the cumbersome transport of the neonates to the computerized tomography (CT) or MRI suite is avoided. In addition, ultrasound is considered a safer modality in the pediatric population due to the lack of 
potential harming effect of ionizing radiation, as in CT, as well as avoiding the need for sedation frequently required for MRI. Ultrasound is the least costly of all cranial imaging modalities and is readily available in all intensive care units. Cranial ultrasound is cheap, safe and can be performed at the cot side by the attending neonatologist or paediatric radiologist.

In this prospective study, we evaluated if the results of the cranial ultrasound, performed during hospitalisation in preterm born infants, may be used by healthcare professionals to predict their developmental outcome 6 months later.

\section{Materials and Methods}

\section{Study population}

Premature infants born before 37 weeks of gestational age (GA) and New Ballard score under 35 admitted into Neonatal Intensive Care Unit of Pediatric Center, Hue Central Hospital, Vietnam, were examined by ultrasound through anterior fontanel during the period from April 2017 to August 2018. This study was approved by the Hue Central Hospital institutional review board. Informed consent was waived by the board.

\section{Ultrasound protocol and findings}

Each infant was prospectively screened by cUS examination for the presence of brain lesions at about 4 and 24 hours and 7 days of age. The anterior and posterior fontanels were commonly used. The mastoid and temporal fontanels were used to obtain axial images for evaluating posterior fossa. The transverse sinus can be examined through posterior fontanel or foramen magnum. SonoSite Sonomax ultrasound equipment with linear 5-7 MHz probe was used. All antiseptic precautions were taken and sterilized ultrasound gel was used. Transvaginal (TV) probe of 7-12 MHz (kept exclusively for cranial ultrasound) was used to evaluate quickly the areas of the brain near convexity. The examination time on an average was 5-7 min and another $1 \mathrm{~min}$ for TV probe to avoid heating effect (if at all it was there). Precaution was taken to avoid undue pressure of the probe on the fontanel. Scans were read by two independent readers aware only of the infants' birth weight and submitted to a third reader in case of disagreement as to the presence or absence or time of onset of a lesion.

cUS diagnoses were categorized as:

1. Intraventricular haemorrhage (IVH)

2. Periventricular leukomalacia (PVL)

3. Ventricular dilatation

4. Other lesion (congenital anomaly, cystic lesion)

\section{Developmental assessment at 6 months}

Families were invited to bring their child for developmental assessment close to the time when he or she would attain 6 months' corrected age. This assessment included the Denver II developmental screening test and a neurologic examination.

\section{Statistical methods}

All the numerical data are expressed as the means \pm standard deviation. The chi-square test or Fisher's exact test was used for the analysis of categorical variables.

To predict of short term developmental outcomes in preterm infants, we compared the US characteristics of the normal and developmental delay groups during the study period. The statistical analysis was conducted using SPSS Statistics version 20 .

\section{Results}

A total of 79 infants born at 24 through 36 weeks of gestation were included in this prospective cohort study. More than onehalf of the entire cohort was male (55.7\%). The mean gestational age $32.1 \pm 3.3$ weeks, and their mean birth weight was $973.7 \pm 315.9 \mathrm{~g}(820-2,250 \mathrm{~g})$. Of these preterm neonates, $54(68.4 \%)$ had normal cUS findings and $25(31.6 \%)$ had abnormal cUS findings. Among the abnormal cUS findings, 15 $(19.0 \%)$ had intraventricular haemorrhage, 7 (8.9\%) had periventricular leukomalacia, $1(1.3 \%)$ had ventricular dilatation, $1(2.5 \%)$ had congenital anomaly and $1(2.5 \%)$ had cystic lesion. All children were performed by the Denver II Developmental Screening Test at the age of 6 months and found $19(24.1 \%)$ cases had developmental delays. There was a significant relationship between abnormal cUS findings (intraventricular haemorrhage, periventricular leukomalacia, ventricular dilatation, other lesion), Apgar score at 5 mins $<7$, hyaline membrane disease, mechanical ventilation and developmental delays (Tables 1 and 2).

Table 1. Assocciation between cUS findings and developmental outcome.

\begin{tabular}{|c|c|c|c|c|}
\hline Characteristics & $\begin{array}{l}\text { Developmental } \\
\text { delays } \\
\mathrm{N}=19\end{array}$ & $\begin{array}{l}\text { Normal } \\
\text { development } \\
\mathrm{N}=60\end{array}$ & $P$ value & $\mathbf{R R}$ \\
\hline $\begin{array}{l}\text { Intraventricular } \\
\text { haemorrhage }\end{array}$ & $8(42.1)$ & $7(11.7)$ & $<0.05$ & 9.6 \\
\hline $\begin{array}{l}\text { Periventricular } \\
\text { leukomalacia }\end{array}$ & $4(21.1)$ & $3(5 \%)$ & $<0.05$ & $\begin{array}{l}10 . \\
3\end{array}$ \\
\hline Ventricular dilatation & $1(5.2 \%)$ & $0(0 \%)$ & $<0.05$ & 18 \\
\hline Congenital anomaly & $1(5.2 \%)$ & $0(0 \%)$ & $<0.05$ & 18 \\
\hline Cystic lesion & $1(5.2 \%)$ & $0(0 \%)$ & $<0.05$ & 18 \\
\hline
\end{tabular}

\section{Discussion}

Periventricular brain damage, whether it is hemorrhagic, ischemic or both, can be associated with abnormalities in developmental outcome [6-8]. Some of the earlier reports focused on infant outcomes associated with isolated germinal matrix hemorrhage (GMH) and/or IVH, but in most cases, infants with such lesions were not disabled [9]. The outcome abnormalities found in GMH and/or IVH with white matter damage, however, can range from subtle cognitive abnormalities to borderline or severe mental retardation 
$[10,11]$. Motor abnormalities are reported often, particularly in association with disabilities such as cerebral palsy [12-15], mental retardation [16], or visual or hearing disturbances [17].

Table 2. Assocciation between newborn characteristics and developmental outcomes.

\begin{tabular}{llll}
\hline Characteristics & $\begin{array}{l}\text { Developmental } \\
\text { delays } \\
\text { N=19 }\end{array}$ & $\begin{array}{l}\text { Normal } \\
\text { development } \\
\text { N=60 }\end{array}$ & P value RR \\
\hline Sex & & & \\
\hline Male & $11(57.9 \%)$ & $33(55.0 \%)$ & $>0.05$ \\
\hline Female & $8(42.1 \%)$ & $27(45.0 \%)$ & \\
\hline
\end{tabular}

\section{GA (week)}

\begin{tabular}{llll}
\hline$<28$ & $2(10.5 \%)$ & $0(0.0 \%)$ & $>0.05$ \\
\hline $28-32$ & $9(47.4 \%)$ & $21(35.0 \%)$ & \\
\hline$\geq 32$ & $8(42.1 \%)$ & $39(65.0 \%)$ & \\
\hline Birth weight & & & \\
\hline$<1500$ & $10(52.6 \%)$ & $17(28.3 \%)$ & $>0.05$ \\
\hline $1500-2499$ & $8(42.1 \%)$ & $40(66.7 \%)$ & \\
\hline$\geq 2500$ & $1(5.3 \%)$ & $3(5.0 \%)$ & \\
\hline
\end{tabular}

\section{Multiple birth}

\begin{tabular}{llll}
\hline Multiple & $4(21.1 \%)$ & $15(25.0 \%)$ & $>0.05$ \\
\hline Singleton & $15(78.9 \%)$ & $45(75.0 \%)$ &
\end{tabular}

\section{Apgar score at $5 \mathrm{~min}$}

\begin{tabular}{|c|c|c|c|c|}
\hline$<7$ & $9(47.4 \%)$ & $14(23.3 \%)$ & $<0.05$ & 2.96 \\
\hline$\geq 7$ & $10(52.6 \%)$ & $46(76.7 \%)$ & & \\
\hline
\end{tabular}

Hyaline membrane disease

\begin{tabular}{lllll}
\hline Yes & $5(26.3 \%)$ & $5(8.3 \%)$ & $<0.05$ & 3.93 \\
\hline No & $14(73.7 \%)$ & $55(91.7 \%)$ & & \\
\hline
\end{tabular}

Mechanical ventilation

\begin{tabular}{lllll}
\hline Yes & $10(52.6 \%)$ & $4(6.7 \%)$ & $<0.05$ & 15.56 \\
\hline No & $9(47.4 \%)$ & $56(93.3 \%)$ & & \\
\hline
\end{tabular}

The sensitivity of cranial ultrasound examinations as predictors of later developmental abnormalities has been reported as $16 \%$ at one and two weeks after birth, increasing to $53 \%$ at six weeks and $58 \%$ if performed when a child is at term-corrected age [18]. The specificity of cranial ultrasound examinations has been $99 \%$ to $100 \%$ in all age groups [18]. The presence of cysts has been reported as a predictor of cerebral palsy, with a sensitivity of $67 \%$ and a specificity of $96 \%$ [13]. In most reports, estimates of the negative predictive value of cranial ultrasound examinations are consistent in that repeated normal examinations predict that an infant is unlikely to have cerebral palsy $[7,13]$.

Our data analysis showed that, 54 (68.4\%) had normal cUS findings and 25 (31.6\%) had abnormal cUS findings. Among the abnormal cUS findings, 15 (19.0\%) had intraventricular haemorrhage, $7(8.9 \%)$ had periventricular leukomalacia, 1 $(1.3 \%)$ had ventricular dilatation, $1(2.5 \%)$ had congenital anomaly and $1(2.5 \%)$ had cystic lesion. Neonatal hypoxic lesions and intracranial hemorrhagic lesions are classified into those that occur in preterm term infants and term neonates. In the former group, these lesions are $\mathrm{GMH}$, intraventricular hemorrhage and PVL [19]. In term infant, hypoxic-ischemic encephalopathy and intracranial hemorrhage are the major manifestations. Sonography is highly accurate in detecting hemorrhage as well as for showing the resulting ventricular dilatation. Similarly, it is the technique of choice in the screening and follow-up of premature neonates for PVL. Multiple sequential examinations are necessary and sonography is ideal in this context.

PVL represents an ischemic injury involving the watershed area of the preterm brain, i.e., the periventricular white matter. There is no accurate way to diagnose PVL in the acute phase. Sonography is the best available imaging modality though it also remains fairly insensitive in early cases. Findings include focal areas of increased echogenicity in the superolateral periventricular areas, most prominent at the level of the atria. Pitfall already mentioned in this context is the normal periventricular flare due to anisotropic effect. Mild cases of PVL can resolve on follow-up sonography (Figure 1). Chronic PVL results in ventriculomegaly, periventricular cystic change, and loss of deep white matter, with the sulci approximating the ventricular wall.

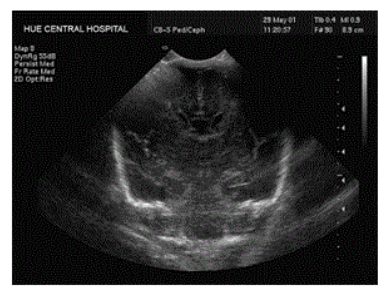

(a)

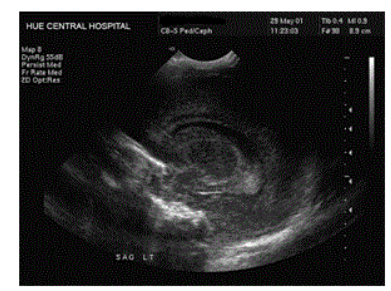

(b)

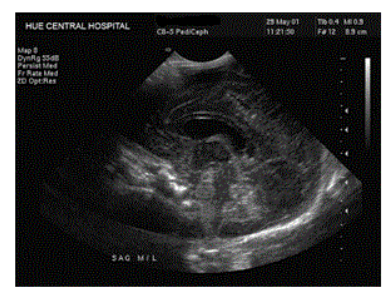

(c)

Figure 1. Periventricular leukomalacia on day 4 in preterm infant born at 29 week GA. (a) Coronal view, (b) Sagittal left view, (c) Midline view.

Most common site of hemorrhage in preterm neonates is a germinal matrix [20]. This is noted as echogenic areas in the caudothalamic groove. The hemorrhage can extend into the ventricles, former resulting in hydrocephalus (Figure 2).

Two contiguous coronal ultrasound images of the brain show the echogenic focus of right intraventricular hemorrhage grade II which involving the right germinal matrix (top image) and 
then extending into the superior portion of the right lateral ventricle without ventricular dilation (bottom image). The echogenic substance filling the inferior portions of both lateral ventricles in the bottom image is the patient's normal choroid plexus.

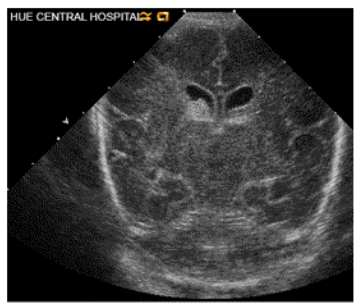

(a)

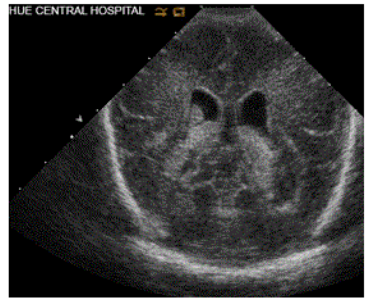

(b)
Figure 2. Ultrasound images of a 2 day old premature infant born at 32 weeks. (a) Echogenic focus of right intraventricular hemorrhage grade II involving the right germinal matrix, (b) Superior portion of the right lateral ventricle without ventricular dilation.

We evaluated the relationship between neonatal cranial ultrasound findings and standardized developmental as sessments in preterm neonates. We found that brain lesions diagnosed by cUS are powerful predictors of developmental delays in this cohort. They are intraventricular haemorrhage (RR 9.6), periventricular leukomalacia (RR 10.3), ventricular dilatation (RR 18), congenital anomaly (RR 18) and cystic lesion (RR 18). Univaniate analysis revealed that several peninatal variables including afgar score at $5 \mathrm{mins}<7$, hyaline membrane disease and mechanical ventilation are each associated with an elevated risk of developmental delays.

O'Shea et al. [21] evaluated associations between ultrasounddefined lesions of the brain and developmental delays at 24 months' corrected age in 1017 children born before the 28th postmenstrual week. Ultrasound abnormalities were more strongly associated with low Psychomotor Development Index than with low Mental Development Index. Children without cranial ultrasound abnormality had the lowest probability $(23 \%$ and $26 \%$ ) of delayed mental or psychomotor development. Moderate/severe ventriculomegaly was associated with a more than fourfold increase in the risk of psychomotor delay and an almost threefold increase in the risk of mental delay. Echolucency was the next best predictor of delayed mental and psychomotor development. The probability of low scores varied with the number of zones involved and with the location of echolucency. At particularly high risk were infants with bilateral cerebellar hemorrhage, co-occurring ventriculomegaly and echolucency bilateral echolucency, or echolucency located posteriorly.

The most important implication of our study is that clinicians can use ultrasound markers of brain damage as predictors of developmental impairment. Children with these markers can be targeted for early intervention to improve developmental outcome. This use of ultrasound is part of the basis for the Practice Parameter for Neuroimaging of the Neonate in 2002 [22], which recommends cranial ultrasound screening for infants born before 30 weeks' gestation, at 7 to 14 days, and again at 36 to 40 weeks

\section{Conclusion}

Cranial ultrasound provides a good screening tool for detection of brain injury in preterm infants during hospitalisation. The result of this examination is used as part of the clinical evaluation and may guide the decision to continue intensive care of the preterm infant, but also to inform parents about their child's prognosis. Future studies are necessary to evaluate whether MRI of preterm infants can lead to a more accurate prediction of the neurodevelopmental impairment.

\section{Acknowledgement}

The authors are grateful to physicians, administrative staff at Neonatal Intensive Care Unit of Hue Central Hospital for allowing us to undertake this research.

\section{Funding}

This work did not receive any funding.

\section{Conflicts of Interest}

The authors declare no conflicts of interest.

\section{Informed Consent}

Informed consent was waived.

\section{Author Contributions}

All participated in the study design, data collection, and literature search. Data was analyzed by TTTQ. TNVA and NHS wrote the paper. All authors read and approved the final manuscript.

\section{Institutional Review Board Approval}

The present study was approved by the Hue Central Hospital Review Board.

\section{Ethical Compliance}

This study was conducted in compliance with the ethical standards of the responsible institution on human subjects as well as with the Helsinki Declaration.

\section{References}

1. Argyropoulou MI. Brain lesions in preterm infants: Initial diagnosis and follow-up. Pediatr Radiol 2010; 40: 811-818.

2. Veyrac C, Couture A, Saguintaah M, Baud C. Brain ultrasonography in the premature infant. Pediatr Radiol 2006; 36: 626-635.

3. Cheong JL, Doyle LW. Increasing rates of prematurity and epidemiology of late preterm birth. J Paediatr Child Health 2012; 48: 784-788. 
4. Purisch SE, Gyamfi-Bannerman C. Epidemiology of preterm birth. Semin Perinatol 2017; 41: 387-391.

5. Vogel JP, Chawanpaiboon S, Moller AB, Watananirun K, Bonet M, and Lumbiganon P. The global epidemiology of preterm birth. Best Pract Res Clin Obstet Gynaecol 2018; 52: 3-12.

6. Levene MI. Is neonatal cerebral ultrasound just for the voyeur? Arch Dis Child 1988; 63: 1-2.

7. Levene MI. Cerebral ultrasound and neurological impairment: Telling the future. Arch Dis Child 1990; 65: 469-471.

8. Tudehope DI. Cranial ultrasonography as a diagnostic and predictive tool in neonatal periventricular haemorrhage. Aust Paediatr J 1985; 21: 249-250.

9. Aziz K, Vickar DB, Sauve RS, Etches PC, Pain KS, and Robertson CM. Province-based study of neurologic disability of children weighing 500 through 1249 grams at birth in relation to neonatal cerebral ultrasound findings. Pediatrics 1995; 95: 837-844.

10. Ross G, Boatright S, Auld PA, and Nass R. Specific cognitive abilities in 2-year-old children with subependymal and mild intraventricular hemorrhage. Brain Cogn 1996; 32: 1-13.

11. Whitaker AH, Feldman JF, Van Rossem R, Schonfeld IS, Pinto-Martin JA, Torre C, Blumenthal SR, and Paneth NS. Neonatal cranial ultrasound abnormalities in low birth weight infants: relation to cognitive outcomes at six years of age. Pediatrics 1996; 98: 719-729.

12. Doyle LW, Betheras FR, Ford GW, Davis NM, and Callanan C. Survival, cranial ultrasound and cerebral palsy in very low birthweight infants: 1980s versus 1990s. J Paediatr Child Health 2000; 36: 7-12.

13. Graham M, Levene MI, Trounce JQ, and Rutter N. Prediction of cerebral palsy in very low birthweight infants: Prospective ultrasound study. Lancet 1987; 2: 593-596.

14. Hesser U, Katz-Salamon M, Mortensson W, Flodmark O, and Forssberg H. Diagnosis of intracranial lesions in verylow-birthweight infants by ultrasound: incidence and association with potential risk factors. Acta Paediatr Suppl 1997; 419: 16-26.

15. Pinto-Martin JA, Riolo S, Cnaan A, Holzman C, Susser MW, and Paneth N. Cranial ultrasound prediction of disabling and nondisabling cerebral palsy at age two in a low birth weight population. Pediatrics 1995; 95: 249-254.
16. Wilkinson I, Bear J, Smith J, Gill A, Challinor C, Jones W, Shahidullah M, and Wooderson S. Neurological outcome of severe cystic periventricular leukomalacia. J Paediatr Child Health 1996; 32: 445-449.

17. Jacobson L, Ek U, Fernell E, Flodmark O, and Broberger U. Visual impairment in preterm children with periventricular leukomalacia-visual, cognitive and neuropaediatric characteristics related to cerebral imaging. Dev Med Child Neurol 1996; 38: 724-735.

18. Nwaesei CG, Allen AC, Vincer MJ, Brown SJ, Stinson DA, Evans JR, and Byrne JM. Effect of timing of cerebral ultrasonography on the prediction of later neurodevelopmental outcome in high-risk preterm infants. J Pediatr 1988; 112: 970-975.

19. Vergani P, Locatelli A, Doria V, Assi F, Paterlini G, Pezzullo JC, and Ghidini A. Intraventricular hemorrhage and periventricular leukomalacia in preterm infants. Obstet Gynecol 2004; 104: 225-231.

20. Burstein J, Papile LA, and Burstein R. Intraventricular hemorrhage and hydrocephalus in premature newborns: A prospective study with CT. AJR Am J Roentgenol 1979; 132: 631-635.

21. O'Shea TM, Kuban KC, Allred EN, Paneth N, Pagano M, Dammann O, Bostic L, Brooklier K, Butler S, Goldstein DJ, Hounshell G, Keller C, McQuiston S, Miller A, Pasternak S, Plesha-Troyke S, Price J, Romano E, Solomon KM, Jacobson A, Westra S, and Leviton A. Neonatal cranial ultrasound lesions and developmental delays at 2 years of age among extremely low gestational age children. Pediatrics 2008; 122: e662-e669.

22. Miller S, Ferriero D, Barkovich AJ, and Silverstein F. Practice parameter: Neuroimaging of the neonate: Report of the quality standards subcommittee of the American academy of neurology and the practice committee of the child neurology society. Neurology 2002; 59: 1663-1664.

\section{*Correspondence to}

Huynh Quang Huy

Department of Radiology

Pham Ngoc Thach University of Medicine

Vietnam 